\title{
LA NATURALEZA Y OBRA DEL ESPÍRITU SEGÚN JUAN 16:12-15
}

\author{
Jared Barrenechea
}

\section{Introducción}

El Espíritu Santo está presente a lo largo de toda la historia bíblica. Aparece en la fundación del mundo (Gn 1:2) y fue, junto con la Deidad, el guiador del pueblo de Israel (Is 63:7-14). Participó activamente en la encarnación del Hijo (Mt 1:18-20) y en el desarrollo de su ministerio (Mc 1:8-12; Lc 3:16, 22; 4:1, 14, 18; 10:21; Jn 1:32, 33). El apóstol Juan registra el momento en que Jesús presenta al Espíritu como su vicario en la tierra (Jn 14-16). Lo señala como "conso-

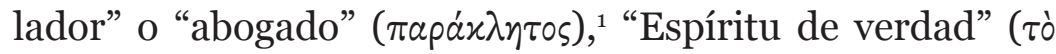

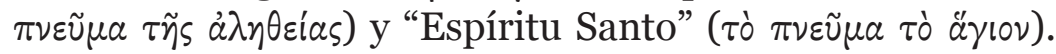
El hecho de que Jesús presente al Espíritu de esta manera demuestra la importancia de su tarea y función para los creyentes de todas las épocas.

Es precisamente la singularidad de la descripción juanina sobre el Parákletos (Jn 14-16), que se distingue de los sinópticos, lo que ha cautivado a muchos teólogos. ${ }^{2}$ Es po-

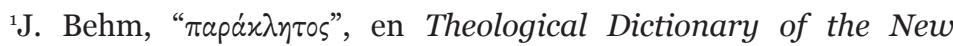
Testament, eds. G. Kittel, G. W. Bromiley y G. Friedrich, vol. 5 (Grand Rapids, MI: Eerdmans,1964), 804. En adelante TDNT.

${ }_{2}$ "No hay pasaje en la Escritura que tenga más información acerca del Parákletos que Jn 16:7-15”. Lawrence J. Lutkemeyer, “The Role of the Paraclete (Jn. 16:7-15)”, Catholic Biblical Quarterly 8, no. 2 (1946): 220. "Cada comentador ha abordado el problema desde Agustín". Véase Donald A. Carson, "The Function of the Paraclete in John 16:7-11", Journal of Biblical Literature 98, no. 4 (1979): 547. 
sible decir, siguiendo a Vincent Taylor, que ese registro del apóstol es "el clímax y corona de la revelación bíblica acerca del Espíritu Santo". ${ }^{3}$ Sin duda, estos textos son una fuente perenne para la comprensión de su naturaleza y función. Es pertinente entonces preguntarse al leerlos: ¿Cuál es la naturaleza y obra del Espíritu Santo en conexión con las otras personas de la Deidad? ¿De qué manera los así llamados dichos Parákletos de Juan aclaran esta cuestión? Más específicamente deseamos preguntarnos, ¿cómo responder a estas preguntas a la luz de Jn 16:12-15?

\section{El texto de Juan 16:12-15 ${ }^{4}$}

El registro de Jn 16:12-15 es el último de los cinco dichos Parákletos (Jn 14:16-17; 14:26; 15:26; 16:7-11) ${ }^{5}$ expresados por Jesús a sus discípulos en el aposento alto. En todo su discurso (Jn 14-16), Jesús destacó la función del Espíritu en relación a él y a favor de la salvación del hombre, al afirmar que "mora con vosotros" (Jn 14:17), "enseñará todas las cosas" (14:26), "dará testimonio de mí" (15:26), "convencerá al mundo, de pecado, justicia y de juicio" (16:8), y "guiará en toda la verdad" (16:13). ${ }^{6}$ Así mismo dijo, en relación al Padre:

3Vincent Taylor, et. al., The Doctrine of the Holy Spirit (London: The Epworth Press, 1938), 66, citado en Amin A. Rodor, "O Espírito-Parákletos No Quarto Evangelho”, Parousia, no. 2 (2005): 53.

${ }^{4}$ Para esta sección se utilizará una traducción propia. Pero para las demás secciones se citará de La Biblia de las Américas, a menos que se indique otra cosa.

5Thomas B. Slater, "The Paraclete as Advocate in the Community of the Beloved Disciple”, African Theological Journal 20, no. 2 (1991): 101.

${ }^{6}$ Véase el cuadro sinóptico de los discursos Parákletos de Jesús presentado en Jerome H. Neyrey, The Gospel of John, New Cambridge Bible Commentary (Cambridge, NY: Cambridge University Press, 2007), 269. 
“y Él os dará otro Consolador" (14:16), "el Padre [lo] enviará en mi nombre" (14:26), "que procede del Padre" (15:26). En todo el discurso se observa una intrínseca relación trinitaria cuya obra se enfoca en los discípulos y en los futuros creyentes (16:13) con propósitos soteriológicos (salvíficos) y escatológicos (hacia la parusía de Cristo, cf. 14:1-3).

En ese contexto, Jn 16:12-15 parece concentrar un mensaje final y urgente para sus discípulos porque Jesús dice: "Todavía tengo muchas cosas que decirles, pero ahora no las pueden llevar" (16:12). El presente indicativo del verbo "tengo" ( $\left.\xi^{\prime} \chi \omega\right)$ señala que Juan entendía que es una acción real en proceso que continuaría el Espíritu Santo. Por eso Jesús manifiesta: "Pero cuando venga él, el Espíritu de Verdad, les guiará en toda la verdad" (16:13). La presencia de "pero cuando" (ö $\tau \alpha \nu ~ \delta \grave{\varepsilon}, 16: 13), 7$ indica que el mensaje y la obra de Jesús en la tierra sería continuado por el Espíritu. Además, el uso continuo de verbos en futuro ${ }^{8}$ señalan que Jesús hace una declaración profética a sus discípulos acerca de la llegada del "Espíritu de verdad" y que la obra de este se proyectaría hasta su Segunda Venida (14:1-3). De este modo les da esperanza de que siempre estaría con ellos a través del Espíritu.

\section{La personalidad y divinidad del Espíritu}

Según Mario Veloso, Juan usa la palabra espíritu $(\pi \nu \varepsilon \tilde{\mu} \mu \alpha)$ con varios significados pero "cuando a pneûma se agregan las palabras hágion o alétheia, se refiere a lo que lla-

${ }^{7}$ Cuando ö $\tau \alpha \nu$ se encuentra con el subjuntivo "casi siempre se refiere a un hecho futuro”. Roberto Hanna, Sintaxis exegética del Nuevo Testamento Griego (El Paso, TX: Mundo Hispano, 2000), 88.

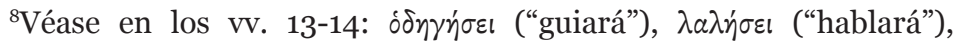

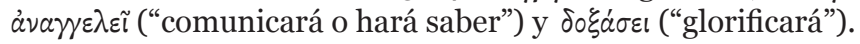


mamos la Tercera Persona de la Divinidad". ${ }^{9}$ Por otro lado, el término paráklētos ( $\pi \alpha \rho a ́ x \lambda \eta \tau o \varsigma){ }^{10}$ significa "uno llamado al lado de" para "ofrecer asistencia en una corte". ${ }^{11}$ También puede significar "consolador", "ayudador" o "auxiliador", "intercesor" y "mediador". ${ }^{12}$ La amplitud semántica de éste término, en palabras de Ignace De la Potterie, designa la "función de asistencia que él ejerce en la tierra a favor de los creyentes" ${ }^{13}$ Ambos términos relacionados entre sí destacan la naturaleza personal del Espíritu. ${ }^{14}$

En su discurso, Jesús promete enviar al "otroparáklētos"

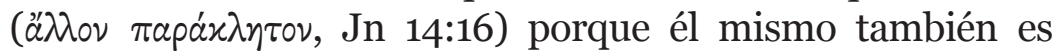

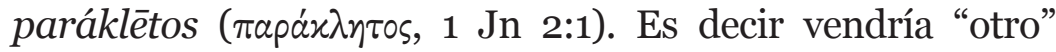

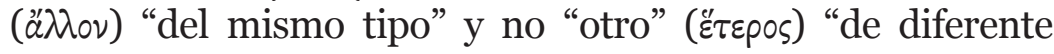

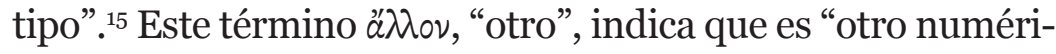

${ }^{9}$ Mario Veloso, El compromiso cristiano (Buenos Aires: Zunino, 1975), 265.

${ }^{10}$ Este término en el NT es registrado 4 veces en Juan y 1 vez en 1 Juan. El $\pi \alpha \rho a ́ x \lambda \eta \tau o s$, en el último discurso de Jesús, "aparece en una diferente capacidad, a saber, como un Guía y Maestro del fiel”. J. H. Bernard, $A$ Critical and Exegetical Commentary on the Gospel According to St. John (Edinburgh: T\&T Clark, 1985), 2:509.

${ }^{11}$ Max Turner, "Holy Spirit", Dictionary of Jesus and the Gospels, ed. Joel B. Green (Leicester: InterVarsity Press, 1992), 349.

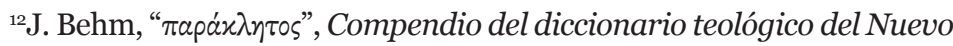
Testamento, eds. Gerhard Kittel, Gerhard Friedrich y Geoffrey W. Bromiley (Grand Rapids, MI: Libros Desafío, 2002), 762; Turner, "Holy Spirit", 349.

${ }^{13}$ Ignace de la Potterie, "Le Paraclet”, La Vie selon l'Esprit: condition du Chrétien (Paris: Les Editions du Cerf, 1965), 85, citado en Veloso, El Compromiso, 268.

${ }^{14}$ En el discurso de Jesús, Juan interrelaciona los términos $\tau \dot{o} \pi \nu \varepsilon \tilde{\mu} \mu \alpha$

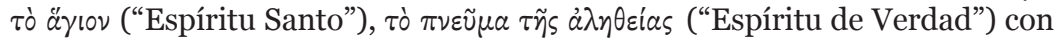
el sustantivo $\pi \alpha \rho a ́ x \lambda \eta \tau o s$ ("Consolador").

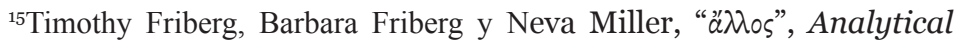
Lexicon of the Greek New Testament (Grand Rapids, MI: Baker, 2000), 
camente distinto, pero similar a él, de su misma naturaleza" ${ }^{16}$ Por así decirlo, otro como Jesús. ${ }^{17}$ Esto pone en evidencia tanto la divinidad como la personalidad del Espíritu.

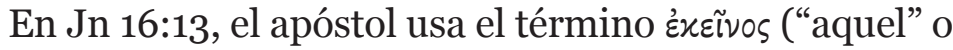
"él"), pronombre demostrativo masculino, para referirse al Espíritu. ${ }^{18}$ Aunque el término $\pi \nu \varepsilon \tilde{u} \mu \alpha$ ("Espíritu”) está en género neutro y algunos traductores infieran que el pronombre debe ser entendido como si se refiriera a un objeto ("ese" o "ello"), el apóstol parece haber usado adrede de forma constante ese pronombre masculino para referirse a la naturaleza personal del Espíritu ("aquel”/“el”). ${ }^{19}$ Además, así como

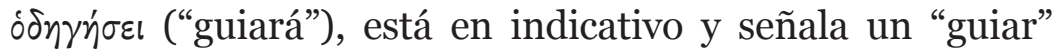
real, los verbos $\lambda \alpha \lambda \eta \dot{\sigma \varepsilon \varepsilon ~(" h a b l a r a ́ ") ~ y ~ a ̉ x o v ́ \sigma \varepsilon l ~(" o i g a ") ~ m u e s-~}$ tran que, para Jesús y Juan, el "hablar” y el "oír” del Espíritu Santo es tan real como las acciones que ellos realizan.

Entre los vv. 13 al 15 se puede ver una interrelación de acciones que exponen la comunión de la Deidad. Jesús afirmó que el Espíritu "no hablará de sí mismo" (16:13) sino "todo lo que oiga" (16:13). Es decir, su obra no sería inde-

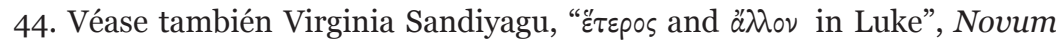
Testamentum 48, no. 2 (2006): 105-30.

${ }^{16}$ Veloso, El compromiso cristiano, 269, 270.

${ }^{17}$ Raymond E. Brown, “The Paraclete”, New Testament Studies 13 (1967): 128, citado por George Eldon Ladd, Teología del Nuevo Testamento, trad. José-María Blanch y Dorcas González Bataller (Barcelona: Clie, 2002), 414. "Sin ser realmente Jesús, Él toma el lugar de Jesús y hace presente a Jesús al mismo tiempo". Johann Joubert, "A Theological Assessment and Interpretation of the Johannine Paraclete-Spirit”, In die Skriflig 41, no. 3 (2007): 510.

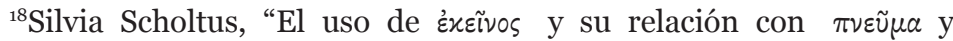

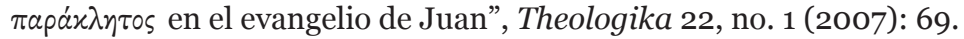

${ }_{19}$ “El Espíritu es pensado en términos personales”. Charles K. Barrett, The Gospel According to St. John: An Introduction with Commentary and Notes on the Greek Text (Philadelphia, PA: Westminster Press, 1978), 482. 
pendiente de la Deidad, sino que actuaría en unidad con el Padre y el Hijo. Por eso declaró: "tomará de lo mío" (16:14) y "todo lo que tiene el Padre es mío" (16:15). Es evidente la reciprocidad entre el Padre y el Hijo, ninguno tiene más que el otro, poseen lo mismo. Esto mismo ocurre en relación con el Espíritu. Aunque Jesús está diciendo “tomará” ( $\lambda \eta \dot{\mu} \mu \varepsilon \tau \tau \iota)$, la forma gramatical ${ }^{20}$ de ese término indica la acción libre y voluntaria del Espíritu en relación al Hijo y al Padre. Esto demuestra igualdad, unidad y propósito en la Deidad.

\section{El Espíritu de Verdad y la Verdad}

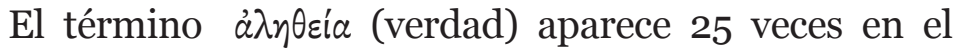
Evangelio de Juan sugiriendo un papel importante en su teología (Jn 1:14, 17; 3:21; 4:23, 24; 5:33; 8:32, 40, 44, 45, 46; $14: 6,17 ; 15: 26 ; 16: 7,13 ; 17: 17,19 ; 18: 37,38)$. Para el apóstol, la verdad no es atemporal ni distante de la realidad humana como en la filosofía griega o gnóstica, ${ }^{21}$ sino que puede ser

${ }^{20} \mathrm{El}$ verbo está en futuro, voz media, modo indicativo. Véase Albert L. Lukaszewski, Mark Dubis y J. Ted Blakley, “Juan 16.14”, The Lexham Syntactic Greek New Testament: SBL Edition (Lexham Press, 2011; Logos Bible Software 7.7), Jn 16:14; La voz media del verbo indica "que el sujeto del verbo está siendo afectado por su propia acción o está actuando sobre sí mismo"; Michael S. Heiser y Vincent M. Setterholm, "Middle”, Glossary of Morpho-Syntactic Terminology (Bellingham, WA: Lexham Press, 2013; Logos Bible Software 7.7). En adelante GMST.

${ }^{21}$ Parménides realiza un contraste entre lo verdadero y lo aparente (Frag. 8.50). Para él lo verdadero es incambiable, inamovible y atemporal (Frag. 1.28-30; 8.1-6; 8.15-19; 8.22-42). Véase, Francis Macdonald Cornford, trad., Plato and Parmenides: Parmenides' Way of Truth and Plato's Parmenides Translated with an Introduction and a Tunning Commentary (London: Trubner \& Co. Ltd., 1939), 30, 35-9, 45; En contraste, Platón, en La República 2.382a-e, aduce que la verdad pertenece a la divinidad y se contrasta con las apariencias o ilusiones. Él dice que "dios es verdad en obra y en palabra, y no cambia ni engaña por medio de ilusiones, discursos o el envío de señales, ya sea en vigilia o sueños”. La República 2.382e. Tomado de Allan 
testificada (Jn $5: 33 ; 18: 37)$, vivida y practicada $(8: 44 ; 1 \mathrm{Jn}$ $1: 8 ; 2: 4,6 ; 3: 8 ; 3 \mathrm{Jn} 4$ ) y es perfectamente cognoscible (Jn 8:32). Esto concuerda con el uso del término hebreo ("verdad") que en el AT no se entiende desde un enfoque dualista sino que es una realidad visible y vivenciable que parte desde Dios y es revelada por Dios al creyente. ${ }^{22}$ Este uso hebraico de $a \lambda \lambda \eta \theta \varepsilon i ́ a$ ("verdad"), que es apoyado por Andreas J. Köstenberger, ${ }^{23}$ Dennis R. Lindsay, ${ }^{24}$ David J. Hawkin e Ignace De la Potterie, ${ }^{25}$ se da de la siguiente manera:

(1) En referencia a Jesús como "lleno de gracia y de

Bloom, The Republic of Plato, 2da ed. (New York: Basic Books, 1991), 61. De este modo Platón sigue a Parménides en colocar la verdad en la atemporalidad (ideas); Charles H. Dodd afirma que a ese término Juan le da un "uso común helenístico" definiéndolo como "la realidad última". Charles H. Dodd, The Interpretation of the Fourth Gospel (Cambridge: Cambridge University Press, 1953), 177. Pero no se toma en cuenta el background hebreo del apóstol Juan.

${ }^{22}$ Para el apóstol Juan la verdad no es un concepto intelectual metafísico abstracto, como la concepción gnóstica o griega, sino que, siguiendo el pensamiento hebreo de emeth (אֶמ = verdad), es un "principio de vida", la Palabra, que demanda "obediencia concreta y acción”. David J. Hawkin, "Revelation and Truth in Johannine Theology", Churchman 116, no. 2 (2002): 107, 110. Además, brinda esperanza escatológica porque "apunta hacia el porvenir, al futuro, a lo que viene". Miguel A. Núñez, "El concepto verdad en sus dimensiones griega y hebrea", Andrews University Seminary Studies 35, no. 1 (1997): 57.

${ }^{23}$ Andreas J. Köstenberger, “'What is Truth?' Pilates Question in Its Johannine and Larger Biblical Context”, Journal of the Evangelical Theological Society 48, no. 1 (2005): 34-5.

${ }^{24}$ Dennis R. Lindsay "What is Truth? 'A $\lambda \eta \theta$ cía in the Gospel of John”, Restoration Quarterly 35, no. 3 (1993): 129-45.

${ }^{25}$ David J. Hawkin, "The Johannine Concept of Truth and Its Implications for a Technological Society", The Evangelical Quarterly 59, no. 1 (1987): 3-13; Hawkin, "Revelation and Truth", 105-12; Ignace de la Potterie, "La Verità in San Giovani”, Rivista Biblica 11 (1963): 5; Ignace de la Potterie, “'Je suis la Voie, la vérité et la Vie' (Jn 14,6)”, Nouvelle Revue Théologique 88, no. 9 (1966): 907-42. 
verdad" (Jn 1:14) y como aquél que imparte esa "gracia y verdad” (Jn 1:17). Sus palabras acerca de su vida, misión y profecía son verdad: "yo digo la verdad" $(8: 45 ; 16: 7)$, "y si digo verdad, ¿̇por qué vosotros no me creéis?” (8:46). Inclusive Juan el Bautista dio "testimonio de la verdad" (5:33). Por último, Jesús afirma ser "el camino, y la verdad, y la vida” y la única vía hacia el Padre (14:6).

(2) Referido a Dios el Padre en cuanto a la forma de adorarlo: "en espíritu y en verdad" (Jn 4:24). Él tiene verdad y de él procede según Jesús: "Os he dicho la verdad que oí de Dios” (8:40). En contraste, según el contexto del texto, Jesús afirma que el diablo "no se ha mantenido en la verdad porque no hay verdad en él” (8:44). Por otro lado, los creyentes son santificados en la Palabra de "verdad" que procede del Padre (17:17). En este sentido, Jesús dijo: "Para esto he venido al mundo, para dar testimonio de la verdad" (18:37).

(3) En referencia directa al Espíritu Santo, Jesús le llama "Espíritu de verdad" (Jn 14:17; 15:26; 16:13) y añade que éste será su testigo $(15: 26 ; 16: 14,15)$.

(4) En referencia al mensaje o Palabra de Dios. En este caso Jesús declara que al permanecer en la Palabra "conoceréis la verdad, y la verdad os hará libres" (8:31-32); “Tu palabra es verdad" (17:17); y Jesús pide al Padre que sus discípulos "sean santificados en la verdad" (17:19) como él se santifica.

(5) En referencia a la relación de la verdad con el creyente, Jesús afirma que la "verdad" puede conocerse (Jn $8: 32)$, vivirse y/o practicarse $(3: 21)^{26}$ y por eso los creyentes pueden ser santificados en esa "verdad" (17:17). ${ }^{27}$

${ }^{26}$ Acerca de la frase "el que practica la verdad" en Jn 3:21, Lindsay comenta que esa expresión ( $\delta \delta \varepsilon \dot{\varepsilon} \pi 0 เ \tilde{\omega} \nu \tau \dot{\eta} \nu a^{2} \lambda \eta^{\prime} \theta \varepsilon ı \alpha$ ) aquí y en 1 Jn 1:6 proviene de la frase veterotestamentaria 'asah emeth que, de acuerdo a Rudolf Bultmann, significa "actuar fielmente". Lindsay, "What is Truth?”, 133, 134.

${ }_{27 “}$ La santificación se produce por la palabra o la verdad que son 
Es posible observar que para el apóstol, la verdad no está separada de Dios (Deidad) sino que Él es la Verdad. Por eso, Jesús, considerando la igualdad del Espíritu Santo en la Dei-

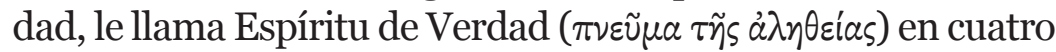
ocasiones (Jn 14:17; 15:26; 16:13; 1 Jn 4:6). Cabe señalar que el

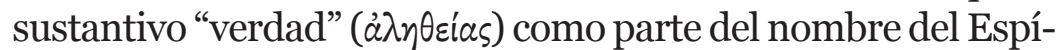
ritu, por su forma genitiva, señala que él es "fuente o poseedor" de verdad..$^{28}$ De este modo, Jesús estaría mostrando: (1) que el Espíritu, así como Él y el Padre son la Verdad; y (2) que su revelación, su Palabra, su profecía (escatología) y su plan de salvación (soteriología) también son la Verdad (17:17).

\section{La obra del Espíritu hacia el creyente}

En Jn 16:12, 13, Jesús anuncia a sus discípulos que su obra será continuada por el Espíritu Santo. Por eso dice: "Pero cuando venga él, el Espíritu de Verdad, les guiará en

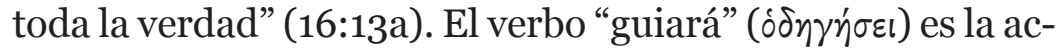
ción principal que indica lo que el Espíritu vendría a realizar por los creyentes. Como ya se ha expuesto lo que se entiende

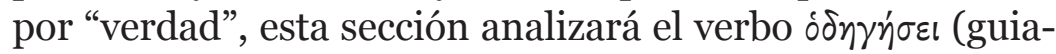
rá) y la preposición $\dot{\varepsilon} \nu$ (en).

(1) El verbo $\delta \delta \eta \gamma \eta \dot{\sigma \varepsilon l, ~ " g u i a r a ́ ” 29 ~(c u y a ~ f o r m a ~ b a ́ s i c a ~}$ es $\delta \delta \eta \gamma \varepsilon ́(\omega)$, literalmente se traduce como "guiar a alguien en el camino" o figurativamente como "instruir", "guiar" y

idénticas". Veloso, El compromiso cristiano, 311. De modo que "el creyente es santificado por el Cristo-Palabra y por el Cristo-Verdad”. Ibíd., 312.

${ }^{28}$ Heiser y Setterholm, "Genitive”, GMST.

${ }^{29}$ Para un estudio más detallado de este término en relación al Evangelio de Juan, véase Jared Barrenechea, "Implicaciones pneumatológicas de

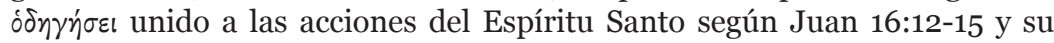
revisión en el panorama histórico-filosófico" (Tesis de Licenciatura, Universidad Peruana Unión, 2015), 76-95. 
"enseñar" ${ }^{30}$ Es interesante notar que este verbo sólo ocurre cinco veces en el NT, de las cuales aparece sólo una vez en el Evangelio de Juan (hapax legomenon). ${ }^{31}$ Esto podría indicar que el apóstol tiene cuidado al usar este término o busca enfatizar una acción muy particular del Espíritu Santo.

Existe la posibilidad de que la iglesia del tiempo de Juan haya estado enfrentando la influencia gnóstica ${ }^{32}$ que consideraba que el guiar (de $\delta \delta \eta \gamma \varepsilon ́ \omega$ ) es una acción "del nous, la mente", 33 la cual "guía a las puertas del conocimiento" $34 \mathrm{o}$ a la "luz del conocimiento". ${ }^{35}$ Esa forma de guiar se podría entender en términos de una epistemología gnóstica dualista, donde la guía es introspección. Quizá por eso Juan utilizó sólo una vez ese verbo con la finalidad de resaltar ante sus lectores, la diferencia del "guiar" del Espíritu. Si observamos el texto, los verbos subordinados a $\delta \delta \eta \gamma \dot{\sigma \varepsilon}$ (guiará), que señalan la forma de cómo sería su acción guiadora, expresan acciones de carácter personal: "hablará" ( $\lambda \alpha \lambda \eta \dot{\sigma \varepsilon l), ~ " o i g a " ~}$

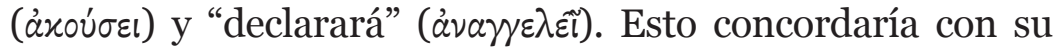

${ }^{30}$ Bern Wilhelm Michaelis, “ódós”, TDNT, 5:97.

${ }^{31}$ En Mt 15:14; Lc 6:39; Jn 16:13; Hch 8:31; Ap 7:17.

${ }^{32}$ Según Giulia Sfameni, ese concepto de gnosis ya se mostraba como un peligro para la Iglesia. Giulia Sfameni Gasparro, "Pablo y los 'primeros gnósticos", Anuario de Historia de la Iglesia 18 (2009): 232. William Combs en su estudio de los escritos gnósticos de Nag Hammadi afirma que "las herejías ya estaban siendo confrontadas en el NT". William W. Combs, "Nag Hammadi, Gnosticism and New Testament Interpretation", Grace Theological Journal 8, no. 2 (1987): 211.

${ }^{33}$ Günter Ebel, “óós`”, en The New International Dictionary of New Testament Theology, ed. Colin Brown (Grand Rapids, MI: Zondervan, 1986), 3:935.

${ }^{34}$ Corpus Hermeticum 7.2, consultado el 18 de enero de 2017, http:// www.hermetics.org/pdf/anotherhermeticum.pdf.

${ }^{35}$ Corpus Hermeticum, 10.21. 
personalidad divina, de manera que su guiar sería una actividad personal del Espíritu y no una actividad introspectiva como en el gnosticismo.

(2) La preposición èv (en) $)^{36}$ indica que "la verdad es la esfera de acción del Parákletos". ${ }^{37}$ En la LXX se prefiere el uso de $\epsilon^{\prime} \nu$ (en) después del verbo ó $\delta \eta \gamma^{2} \omega$ (guiar) ${ }^{38}$ señalando no sólo esfera de acción sino también dirección, ${ }^{39}$ que en el caso del

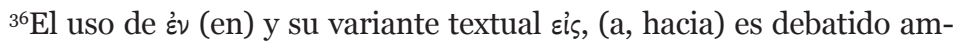
pliamente por los eruditos. Apoyan ह่v (en): Raymond E. Brown, The Gospel according to John XIII-XXI, The Anchor Bible 29, eds. W. F. Albright y D. N. Freedman (New York: Doubleday, 1970), 707; Francis J. Moloney, El Evangelio de Juan, trad. José P. Escobar (Navarra: Verbo Divino, 2005), 451; Barrett, The Gospel According to St. John, 489; George R. Beasley-Murray, John, Word Biblical Commentary 36, ed. David A. Hubbard (Texas, TX: Word Books, 1987), 283. Otros apoyan zis, (a, hacia) porque indica que "el movimiento terminará en el interior del lugar al cual es dirigido”. Ignace de la Potterie citado por Brown, The Gospel according to John XIII-XXI, 707; también Bernard, Gospel According to St. John, 2:509-10; Leon Morris, The Gospel According to John, The New International Commentary on the New Testament, ed. Gordon Fee (Grand Rapids, MI: Eerdmans, 1995), 620-1; Rudolf Bultmann, The Gospel of John: A Commentary (Philadelphia: Westminster Press, 1971), 573.

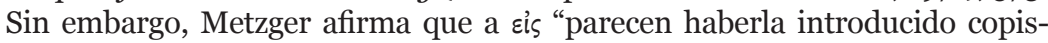
tas que la consideraron más idiomática" que év. Bruce M. Metzger, A Textual Commentary on the Greek New Testament, 2da ed. (New York: United Bible Societies, 1994), 210. Para un estudio más amplio de esta variante textual, véase Barrenechea, "Implicaciones pneumatológicas", 63-8.

${ }^{37}$ Brown, The Gospel according to John XIII-XXI, 707.

${ }^{38} \mathrm{En}$ la LXX, de las 45 veces que aparece el verbo $\delta \delta \eta \gamma \varepsilon ́ \omega, 20$ veces es seguido de la preposición dativa $\epsilon \nu ; 23$ veces por alguna conjunción $u$ otra

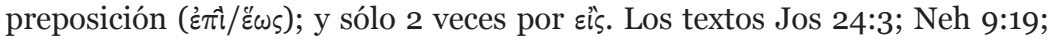
Sal 85:11; 142:10; $138: 24$ proveen clara similitud de forma y sentido en el uso de $€ v$ respecto a Jn 16:13a. Los más claros ejemplos son los dos últimos: Sal 142:10 ("tu buen Espíritu me guíe a [' $\epsilon$ ] tierra de rectitud") que señala extensión espacial; y Sal 138:24 ("y guíame en ['€v] el camino eterno"), que refiere un rumbo dentro de una posición espacial hacia una extensión espacial.

${ }^{39}$ Según Young tanto eis como é $\nu$, que expresan extensión espacial y posición espacial respectivamente, pueden también, en algunas ocasiones, ser

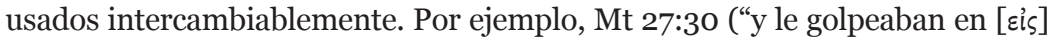


presente estudio, sería referido al guiar del Espíritu.

La obra guiadora del Espíritu hacia el creyente, en base a lo dicho previamente, se podría explicar en términos personales sin limitar su naturaleza divina. Sin ir más allá de lo que el texto muestra, el Espíritu guiaría al creyente "en" (¿่v) todo el ámbito de la Verdad revelada, su Palabra, y sin salirse

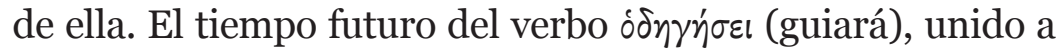
la preposición $\varepsilon^{2} \nu$ (en), expresan de forma implícita, el ámbito y la dirección de la acción del Espíritu: guiaría en la verdad (esfera de acción) y hacia toda la verdad (dirección).

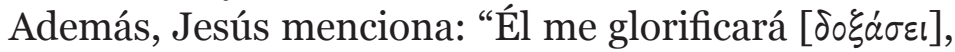
porque tomará de lo mío y les comunicará” (16:14). Esta expresión se complementa con la explicación del párrafo anterior. El Espíritu, al realizar su obra guiadora en el creyente, glorificaría a Cristo. Él tomaría de Cristo ("la Verdad", Jn 14:6; traducción propia), de toda su revelación a lo largo de la historia bíblica, y guiaría en todo el ámbito de esa revelación que es (1) soteriológica (salvífica, cf. Jn 14:26; 16:711), para que los creyentes "sean consagrados por medio de la verdad” (Jn 17:19; traducción propia), y (2) escatológica, orientada hacia la promesa de la segunda venida de Jesús (cf. Jn 14:1-3; 17:24). De esta manera, los creyentes serían conducidos a una unidad salvífica en Cristo. Una unidad que se evidencia en la vida de los creyentes, porque practican y viven la verdad (Jn 16:8).

\section{El Espíritu y su obra profética}

Los tiempos verbales referidos al Espíritu Santo en Jn 16:12-15 expresan su futuro ministerio a favor de los creyen-

la cabeza") y Jn 5:4 ("un ángel descendía de vez en cuando al ['€ $\nu$ ] estanque"). Véase Richard A. Young, Intermediate New Testament Greek: A Linguistic and Exegetical Approach (Nashville, TN: Broadman \& Holman, 1994), 93, 96. 
tes. Pero debe recordarse que el Espíritu estuvo presente en la tierra desde el mismo origen del mundo ( $\mathrm{Gn}$ 1:2). Lo que Jesús hizo es presentarlo en su función vicaria en el contexto del plan salvífico de la Deidad. Así también es importante comprender que fue activo partícipe en el proceso de comunicación de Dios a los profetas a lo largo de todo el AT (cf. $2 \mathrm{~S}$ 23:2, 3; Neh 9:20, 30; Ez 2:2; 3:12, 14, 24; 8:3; 37:1) y también estaría con el Mesías (Is 11:2; 61:1-3). Ese marco amplio que la Biblia muestra, que por razones de espacio no podemos detallar, ayuda a ver que la función profética del Espíritu no es una nueva faceta o nueva forma de comunicación de parte de la Deidad, sino que ya estaba operando en la conducción de su pueblo.

Con eso en mente se deben observar las palabras de Jesús. Él dijo a sus discípulos acerca del Espíritu: "les de-

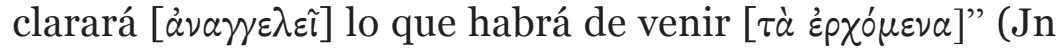
16:13; traducción propia). En los siguientes versículos re-

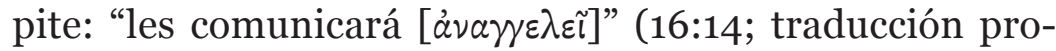

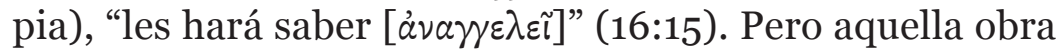
del Espíritu sería a partir de "todo lo que oiga" (16:13) y de lo que "tomara” del Hijo (16:14) y del Padre (16:15). Esta dinámica conjunta de la comunicación divina, aunque no de forma explícita, ya se observa desde el AT (cf. 1 S 10:6, 9, 10). El Espíritu no actuaría de forma independiente sino en perfecta comunión con los otros miembros de la Deidad. Él continuaría con la obra y la revelación de Cristo en la tierra. Pero no traería nuevas revelaciones que no se construyeran sobre la plataforma de la verdad revelada en Cristo $^{40}$ porque su propósito es guiar en esa Verdad.

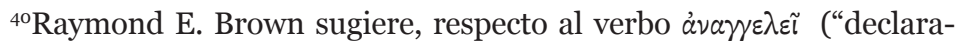
rá”), que "el prefijo ana- tiene el valor del castellano 're': 're-anunciar', 'repetir" o también re-declarar. Esto implicaría "claramente una interpretación o

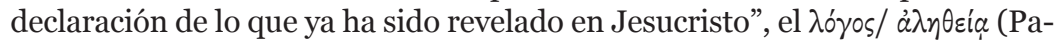
labra/Verdad) hecho carne. Véase Raymond E. Brown, El Evangelio según Juan XIII-XXI (Madrid: Ediciones Cristiandad, 1979), 969. 


\section{Conclusión}

Sin duda el discurso de Jesús sobre el Parákletos es magistral y único. No hay en toda la Biblia una revelación tan amplia sobre el Espíritu Santo. Del estudio de Jn 16:1215 es posible destacar, respecto a la naturaleza y obra del Espíritu, lo siguiente:

(1) El Espíritu Santo es Dios tanto como lo es el Padre y el Hijo. (a) Los tres actúan de forma conjunta y no de forma independiente. (b) Comparten la misma misión salvífica y escatológica a favor de la humanidad. (c) Poseen la Verdad y son la fuente de la Verdad que han revelado a los profetas. (d) El Espíritu Santo es el representante de Cristo.

(2) Al igual que el resto de la divinidad, (a) el Espíritu Santo es una realidad histórica así como sus acciones. (b) Como persona divina, el Espíritu Santo tiene pensamiento y voluntad para actuar en su relación trinitaria y en su misión a favor del creyente. (c) Además, el Espíritu Santo es relacionalmente cognoscible, así como los otros miembros de la Deidad

(3) La obra del Espíritu Santo en Jn 16:12-15 es (a) guiar al creyente en toda la Verdad. Esa Verdad es la Palabra revelada de la Deidad. Su forma de guiar, hablando, oyendo, comunicando o declarando, puede definirse en patrones personales. (b) Al guiar en la Verdad anunciaría lo que habría de venir, es decir, la actividad profética no cesaría, pero esta se construiría en esa Verdad. Y al hacer todo eso (c) glorificaría a Cristo delante de los creyentes conduciéndolos salvíficamente a Cristo y escatológicamente hacia su segunda venida. 
Jared Barrenechea jared.barrenechea@novotempo.com Red Nuevo Tiempo, División Sudamericana

Brasilia, Brasil

Recibido: 30/01/2017 Aceptado: 03/03/2017 